OPEN ACCESS

Edited by:

Hidenori Fukuoka,

Kobe University, Japan

Reviewed by:

Masaaki Yamamoto,

Kobe University Hospital, Japan

Anton Luger,

Medical University of Vienna, Austria

*Correspondence:

Fabio Bioletto

fabio.bioletto@unito.it orcid.org/0000-0001-7550-7023

Specialty section:

This article was submitted to

Pituitary Endocrinology,

a section of the journal

Frontiers in Endocrinology

Received: 07 July 2021 Accepted: 06 September 2021 Published: 24 September 2021

Citation:

Bioletto F, Parasiliti-Caprino M, Berton AM, Prencipe N, Cambria V, Ghigo E, Grottoli $S$ and Gasco V (2021) Development and Internal Validation of a Predictive Model for Adult GH Deficiency

Prior to Stimulation Tests.

Front. Endocrinol. 12:737947. doi: 10.3389/fendo.2021.737947

\section{Development and Internal Validation of a Predictive Model for Adult GH Deficiency Prior to Stimulation Tests}

\author{
Fabio Bioletto *, Mirko Parasiliti-Caprino, Alessandro Maria Berton, Nunzia Prencipe, \\ Valeria Cambria, Ezio Ghigo, Silvia Grottoli and Valentina Gasco
}

Division of Endocrinology, Diabetology and Metabolism, Department of Medical Sciences, University of Turin, Turin, Italy

Background: The diagnosis of adult $\mathrm{GH}$ deficiency (GHD) relies on a reduced $\mathrm{GH}$ response to provocative tests. Their diagnostic accuracy, however, is not perfect, and a reliable estimation of pre-test GHD probability could be helpful for a better interpretation of their results.

Methods: Eighty patients showing concordant $\mathrm{GH}$ response to two provocative tests, i.e. the insulin tolerance test and the GHRH + arginine test, were enrolled. Data on IGF-I values and on the presence/absence of other pituitary deficits were collected and integrated for the estimation of GHD probability prior to stimulation tests.

Results: An independent statistically significant association with the diagnosis of GHD was found both for IGF-I SDS (OR 0.34, 95\%-Cl 0.18-0.65, $\mathrm{p}=0.001$ ) and for the presence of other pituitary deficits (OR 6.55, 95\%-Cl 2.06-20.83, $\mathrm{p}=0.001)$. A low $(<25 \%)$ pre-test GHD probability could be predicted when IGF-I SDS $>+0.91$ in the presence of other pituitary deficits or IGF-I SDS > -0.52 in the absence of other pituitary deficits. A high (>75\%) pre-test GHD probability could be predicted when IGF-I SDS < -0.82 in the presence of other pituitary deficits or IGF-I SDS $<-2.26$ in the absence of other pituitary deficits.

Conclusion: This is the first study that proposes a quantitative estimation of GHD probability prior to stimulation tests. Our risk class stratification represents a simple tool that could be adopted for a Bayesian interpretation of stimulation test results, selecting patients who may benefit from a second stimulation test and possibly reducing the risk of wrong GHD diagnosis.

Keywords: growth hormone deficiency, hypopituitarism, IGF-I, pre-test probability, predictive model

\section{INTRODUCTION}

Adult GH deficiency (GHD) is a heterogeneous disorder that may result from a variety of causes, including structural lesions, genetic abnormalities, traumas, infiltrative diseases, surgery or irradiation to the pituitary gland and/or hypothalamus (1-5). This condition is characterized by altered body composition, glucose intolerance, abnormal lipid profiles, premature atherosclerosis, osteoporosis, impaired quality of life, and increased mortality (6-9). GH replacement treatment 
improves most of these abnormalities (10-14); however, due to the high cost of GH therapy $(14,15)$ and to the concerns of potential long-term risks, particularly the development of diabetes mellitus or malignancies, it is crucial to establish a correct diagnosis in order to offer GH replacement to truly GHdeficient adults $(1,12,14)$.

Adult GHD diagnosis depends on the demonstration of a subnormal rise in peak serum GH level in response to one or more GH stimulation tests $(1,16-18)$. The most relevant stimulation tests to this scope are the insulin tolerance test (ITT), the GH-releasing-hormone + arginine (GHRH + ARG) test, the GHRH + GH-releasing-peptide-6 (GHRH + GHRP-6) test, and the glucagon test $(1,16-18)$. Their accuracy for GHD diagnosis, though high, is not perfect. Their estimated sensitivity ranges from $87 \%$ to $96 \%$, while their estimated specificity from $79 \%$ to $92 \%$, depending on the considered study (19-22). This can make the recognition of GHD challenging, and may possibly result in a wrong diagnosis. This picture is further complicated by the interaction between GHD and body mass index (BMI); in fact, obesity is a state of functional relative reduction of $\mathrm{GH}$ production, both in terms of spontaneous secretion (23-25) and in terms of responsiveness to stimulation tests $(26,27)$. To mitigate this issue, specific BMI-related cut-offs have been proposed for most GH provocative tests (20-22), including lately the ITT (28), but false-negative and false-positive results may still occur.

In light of this, the results of a stimulation test should not be interpreted as an unquestionable dichotomous answer on patient's diagnosis. On the contrary, it should be encouraged to think to the process of GHD diagnosis through a Bayesian approach, in which any additional test result modifies (upward or downward) the probability that a given patient has GHD, as already discussed and suggested for many other medical and endocrinological conditions (29-33). This Bayesian approach to the GHD diagnostic work-up is methodologically well-grounded and allows a more efficient handling and interpretation of stimulation test results, but poses a great problem, i.e. a reliable quantitative estimate of pre-test GHD probability based on other presenting features.

As reported in literature and in current guidelines (1, 34-36), the two most important parameters predicting a final diagnosis of GHD are the presence/absence of other pituitary deficits and the IGF-I values, considered in terms of age-specific standard deviation score (SDS). The information that can be deduced from these features might thus be seen as a proxy for the estimation of GHD probability prior to stimulation tests. Up to date, however, there is no shared, accurate and standardized model that quantitatively estimates this risk. The aim of this study was to develop and internally validate such a model.

\section{METHODS}

\section{Patient Selection}

Data of all patients with a history of pituitary disease who underwent two different stimulation tests for the evaluation of
GH secretion at the Neuroendocrinology Clinic of our Center between January 2017 and January 2019 were collected from prospective registries and analyzed retrospectively.

The two stimulation tests used for the dynamic evaluation of GH response were the ITT and the GHRH + ARG test. ITT was performed by intravenous injection of $0.1-0.15 \mathrm{IU} / \mathrm{kg}$ of regular insulin at $0 \mathrm{~min}$ in normal weight and overweight/obese subjects, respectively; blood sampling for $\mathrm{GH}$ was performed every $15 \mathrm{~min}$ from 0 to $+90 \mathrm{~min}$; adequate hypoglycemia was defined as the achievement of a glucose level $<40 \mathrm{mg} / \mathrm{dl}$. GHRH + ARG test was performed by intravenous injection of $1 \mu \mathrm{g} / \mathrm{kg}$ of GHRH at $0 \mathrm{~min}$ and of $0.5 \mathrm{~g} / \mathrm{kg}$ of ARG from 0 to $+30 \mathrm{~min}$; blood sampling for $\mathrm{GH}$ was performed every $15 \mathrm{~min}$ from +30 to $+60 \mathrm{~min}$. All subjects underwent the two testing sessions in random order and at least 3 days apart. In the case of patients with functional pituitary adenomas, all tests for the assessment of GH deficiency were conducted after achieving the cure of the underlying pathology. Similarly, no tests were performed in patients presenting other potential interferents with the functional evaluation of GH/IGF-I axis (e.g., catabolic states, liver cirrhosis, end-stage chronic kidney disease, altered and uncorrected thyroid function, exogenous glucocorticoid treatment other than replacement therapy).

Approval from local ethics committees was obtained for the analysis of patient data. Written informed consent was obtained from all included patients.

\section{Data Collection}

For each patient, all the following data were collected: gender, age, BMI, IGF-I values, presence/absence of other pituitary deficiencies apart from GHD, peak GH value at ITT, peak GH value at GHRH + ARG test. The diagnosis of other pituitary deficiencies was made according to current international guidelines (37). The GH response to GHRH + ARG test was defined as normal when $\mathrm{GH}>11.0 \mu \mathrm{g} / \mathrm{l}$ for lean subjects, when $\mathrm{GH}>8.0 \mu \mathrm{g} / \mathrm{l}$ for overweight subjects and when $\mathrm{GH}>4.0 \mu \mathrm{g} / \mathrm{l}$ for obese subjects $(1,21)$. The $\mathrm{GH}$ response to ITT was defined as normal when $\mathrm{GH}>3.5 \mu \mathrm{g} / \mathrm{l}$ for lean subjects and when $\mathrm{GH}>1.3$ $\mu \mathrm{g} / \mathrm{l}$ for overweight or obese subjects, in agreement with a recent paper that has identified these as the best BMI-related GH cutoffs for the diagnosis of GHD at ITT (28). Patients with a concordantly normal response to both tests were considered as having a normal function of the somatotroph axis. Patients with concordantly deficient response to both tests were considered as being affected by GHD.

\section{Analytical Methods}

Serum GH levels $(\mu \mathrm{g} / \mathrm{l})$ were measured in duplicate by IRMA method (IRMA GH, Beckman Coulter, Czech Republic). The sensitivity of the assay was $0.033 \mu \mathrm{g} / \mathrm{l}$. The inter- and intra-assay coefficients of variation (CV) were $9.0-14.0 \%$ and $2.4-6.5 \%$, respectively. Serum IGF-I levels $(\mu \mathrm{g} / \mathrm{l})$ were measured in duplicate by RIA method (SM-C-RIA-CT, DIAsource ImmunoAssays, Belgium) after acid-ethanol extraction to avoid interference by binding proteins. The sensitivity of the method was $0.25 \mu \mathrm{g} / \mathrm{l}$. The inter- and intra-assay CV were $6.8-14.9 \%$ and 4.5-7.0\%, respectively. IGF-I levels are expressed both as an 
absolute value and as SDS. The SDS was calculated for each subject, in accordance with the published normality data on a population of 547 healthy Italian subjects (38), as the difference between patient's IGF-I value and age-specific mean IGF-I value, divided by age-specific IGF-I standard deviation. All other biochemical variables were assayed in plasma or serum using standard methods.

\section{Statistical Analysis}

Baseline characteristics of all patients included in the analysis are summarized using mean and standard deviation for continuous variables and percent values for categorical data. Between-group differences were evaluated by Student t-test for continuous variables, and by either chi-squared test or Fisher's exact test for categorical variables, as appropriate.

A multivariate logistic regression model was used to examine the predictive performance of IGF-I SDS and presence/absence of other pituitary deficits for the final diagnosis of GHD. In order to reduce the potential bias deriving from class imbalance, undersampling of the majority class was adopted to achieve a 1:1 ratio between patients with and without GHD (39). Randomness in estimates was reduced by iterating the undersampling process ten times and averaging the retrieved coefficients. Model calibration was evaluated at each iteration by the Hosmer-Lemeshow test. Final model performance was evaluated on the original dataset by the area under curve (AUC) at Receiver Operating Characteristic (ROC) analysis. A ten-fold cross-validation algorithm was adopted for internal validation, in order to provide an estimate of model performance on unseen data. After a random split of the original sample into ten groups, the described modeling process was entirely repeated in nine of them, and its performance was evaluated in the tenth. The process was then repeated ten times, rotating the validation group at each round. Final model performance was obtained as the average performance over the ten iterations.

A p-value $<0.05$ was adopted for the definition of statistical significance. Statistical analysis was performed using STATA 16 (StataCorp, College Station, Texas, USA).

\section{RESULTS}

\section{General Characteristics of the Study Population}

One hundred and twenty-three patients underwent both ITT and GHRH + ARG test in our Center between January 2017 and January 2019, in the appropriate clinical context for the suspicion of GHD according to the international guidelines (1). Of these, 3 patients were excluded because of the lack of informed consent, and 14 patients were excluded due to the non-achievement of adequate hypoglycemia (glucose $<40 \mathrm{mg} / \mathrm{dl}$ ) during ITT. Among the remaining 106 patients, 26 were excluded due to discordant results between ITT and GHRH + ARG test. Therefore, finally, 80 patients were included in our final analysis. In 24 of these $(30.0 \%)$, both stimulation tests concordantly showed a normal
$\mathrm{GH}$ response; these patients were thus considered as having a normally functioning somatotroph axis. In the remaining 56 (70.0\%), both stimulation tests concordantly showed a deficient $\mathrm{GH}$ response; these patients were thus considered as having GHD.

Patients with GHD were slightly older than those without GHD $(49.9 \pm 11.8$ vs $43.3 \pm 14.8$ years, $\mathrm{p}=0.032)$; no difference between the two groups could be noted in terms of gender $(37.0 \%$ vs $50.0 \%$ females, $\mathrm{p}=0.270$ ). BMI was similar in the two groups, both when expressed as a continuous measure $(26.7 \pm 5.2$ vs $25.1 \pm$ $\left.5.8 \mathrm{~kg} / \mathrm{m}^{2}, \mathrm{p}=0.216\right)$ and when expressed in terms of weight category $(\mathrm{p}=0.807)$. Other pituitary deficits were more common in patients with GHD than in those without GHD $(72.2 \%$ vs $30.8 \%, \mathrm{p}<0.001)$. IGF-I levels were lower in patients with GHD than in those without GHD, both when expressed in terms of absolute values $(90.0 \pm 43.7$ vs $176.6 \pm 73.2 \mu \mathrm{g} / \mathrm{l}, \mathrm{p}<0.001)$ and in terms of SDS $(-1.03 \pm 0.90 v s-0.16 \pm 0.89, \mathrm{p}<0.001)$. No significant differences between the two groups were found in terms of pituitary pathology $(p=0.272)$. Clinical characteristics of these two groups are summarized in Table 1.

\section{Model Construction and Internal Validation}

A clinical prediction model for the diagnosis of GHD was constructed by multivariate logistic regression. The variables included in the model, chosen according to data from the existing literature, were IGF-I SDS and the presence/absence of other pituitary deficits. An independent statistically significant association with the diagnosis of GHD was found both for IGF-I SDS (OR 0.34, 95\%-CI 0.18-0.65, $\mathrm{p}=0.001$ ) and for the presence of other pituitary deficits (OR 6.55, 95\%-CI 2.06-20.83, $\mathrm{p}=0.001$ ) (Table 2).

In order to reduce class imbalance, a random undersampling of the majority class was performed and iterated ten-times. Final model parameters were retrieved through averaging of regression coefficients over these ten iterations. This allowed the calculation of the probability (P) of GHD according to the following formula: $\mathrm{P}=\mathrm{e}^{\mathrm{z}} /\left(1+\mathrm{e}^{\mathrm{z}}\right)$, where $\mathrm{z}=1.82$ * other pituitary deficits (yes $=1$, no $=0$ ) -1.27 * IGF-I SDS -1.77 . The HosmerLemeshow test did not reveal any significant miscalibration $(\mathrm{p}>$ 0.30 in all iterations).

The predictive performance of this model was assessed on the original dataset by the calculation of the AUC at ROC analysis, which was equal to 0.826 (Figure 1). Notably, the model performance was comparable in lean subjects (AUC 0.849) and in overweight/obese subjects (AUC 0.816). Internal validation of the model was performed through ten-fold cross-validation, as already described. The final estimation of the model performance on unseen data, obtained as the average AUC over the ten iterations, was equal to 0.820 , thus reassuring about a substantially null overfitting effect.

\section{Risk Class Stratification}

In order to simplify the use of the model in clinical practice, the equation of the logistic regression model was used to retrieve GHD probabilities estimated according to the considered predictive variables. Results were stratified according to the presence or the absence of other pituitary deficits and for each case the cut-offs of IGF-I SDS predicting a probability of GHD of 
TABLE 1 | Baseline clinical characteristics in patients diagnosed with and without GHD.

\begin{tabular}{|c|c|c|c|}
\hline Variables/parameters & No GHD (n = 26) & GHD ( $n=54)$ & p-value \\
\hline Age (years); mean \pm SD & $43.3 \pm 14.8$ & $49.9 \pm 11.8$ & 0.032 \\
\hline Female sex; $\mathrm{n}(\%)$ & $13(50.0)$ & $20(37.0)$ & 0.270 \\
\hline IGF-I $(\mu \mathrm{g} / \mathrm{l}) ;$ mean $\pm \mathrm{SD}$ & $176.6 \pm 73.2$ & $90.0 \pm 43.7$ & $<0.001$ \\
\hline IGF-I SDS; mean \pm SD & $-0.16 \pm 0.89$ & $-1.03 \pm 0.90$ & $<0.001$ \\
\hline Presence of other pituitary deficiencies; n (\%) & $8(30.8)$ & $39(72.2)$ & $<0.001$ \\
\hline Number of other pituitary deficiencies; n (\%) & & & 0.002 \\
\hline None & $18(69.2)$ & $15(27.8)$ & \\
\hline One & $5(19.2)$ & $11(20.4)$ & \\
\hline Two & $1(3.9)$ & $11(20.4)$ & \\
\hline Three or more & $2(7.7)$ & $17(31.4)$ & \\
\hline \multicolumn{4}{|l|}{ Prevalence of other specific pituitary deficiencies } \\
\hline HPA axis deficiency; $\mathrm{n}(\%)$ & 5 (19.3) & $29(53.7)$ & 0.003 \\
\hline HPG axis deficiency; n (\%) & $4(15.4)$ & $28(51.9)$ & 0.002 \\
\hline HPT axis deficiency; $n$ (\%) & $4(15.4)$ & $27(50.0)$ & 0.003 \\
\hline ADH deficiency; $n$ (\%) & $1(3.9)$ & $2(3.7)$ & $0.698^{a}$ \\
\hline BMl $\left(\mathrm{kg} / \mathrm{m}^{2}\right) ;$ mean $\pm \mathrm{SD}$ & $25.1 \pm 5.8$ & $26.7 \pm 5.2$ & 0.216 \\
\hline BMl category; n (\%) & & & 0.807 \\
\hline Normal $\left(\mathrm{BMl}<25 \mathrm{~kg} / \mathrm{m}^{2}\right)$ & $12(46.2)$ & $21(38.9)$ & \\
\hline Overweight (BMl $\geq 25 \mathrm{~kg} / \mathrm{m}^{2}$ and $<30 \mathrm{~kg} / \mathrm{m}^{2}$ ) & $8(30.8)$ & $20(37.0)$ & \\
\hline Obese $\left(\mathrm{BMl} \geq 30 \mathrm{~kg} / \mathrm{m}^{2}\right)$ & $6(23.1)$ & $13(24.1)$ & \\
\hline Pituitary disease; n (\%) & & & 0.272 \\
\hline Sellar mass & $22(84.6)$ & $37(68.5)$ & \\
\hline Empty sella (primary or secondary) & $1(3.8)$ & $7(13.0)$ & \\
\hline Other & $3(11.6)$ & $10(18.5)$ & \\
\hline \multicolumn{4}{|l|}{$\begin{array}{l}\text { (Pituitary hypoplasia, Idiopathic } \\
\text { hypopituitarism, Traumatic brain injury) }\end{array}$} \\
\hline Type of sellar mass; $n(\%)^{b}$ & & & $0.253^{\mathrm{a}}$ \\
\hline Non-functioning adenoma & $14(63.7)$ & $24(64.9)$ & \\
\hline PRL-secreting adenoma & $6(27.3)$ & $6(16.2)$ & \\
\hline GH-secreting adenoma & $0(0.0)$ & $2(5.4)$ & \\
\hline ACTH-secreting adenoma & $1(4.5)$ & $0(0.0)$ & \\
\hline TSH-secreting adenoma & $1(4.5)$ & $0(0.0)$ & \\
\hline Craniopharyngioma & $0(0.0)$ & $3(8.1)$ & \\
\hline Rathke's cleft cyst & $0(0.0)$ & $2(5.4)$ & \\
\hline
\end{tabular}

${ }^{a} p$-value at Fisher's exact test.

${ }^{b}$ Percentages calculated with respect to the total number of patients presenting a sellar mass (i.e., 22 patients among those without GHD and 37 patients among those with GHD). $A C T H$, adrenocorticotropic hormone; $A D H$, anti-diuretic hormone; BMl, body mass index; GH, growth hormone; GHD, growth hormone deficiency; HPA, hypothalamus-pituitary-adrenal; HPG, hypothalamus-pituitary-gonads; HPT, hypothalamus-pituitary-thyroid; IGF-I, insulin-like growth factor I; PRL, prolactin; SD, standard deviation; SDS, standard deviation score; TSH, thyroid-stimulating hormone.

TABLE 2 | GHD prediction by multivariate logistic regression on the full dataset.

\begin{tabular}{|c|c|c|c|}
\hline Predictor & OR & $95 \% \mathrm{Cl}$ & p-value \\
\hline IGF-I SDS & 0.34 & $0.18-0.65$ & 0.001 \\
\hline Presence of other pituitary deficiencies & 6.55 & $2.06-20.83$ & 0.001 \\
\hline
\end{tabular}

Cl, confidence interval; GHD, growth hormone deficiency; IGF-I, insulin-like growth factor I; OR, odds ratio; SDS, standard deviation score.

$25 \%, 50 \%$ and $75 \%$ were retrieved. In the absence of other pituitary deficits, these cut-offs were $-0.52,-1.39$ and -2.26 , respectively. In the presence of other pituitary deficits, these cut-offs were $+0.91,+0.04$ and -0.82 , respectively (Table 3 ).

\section{DISCUSSION}

In this study, we developed and internally validated a multivariate prediction model for the estimation of GHD probability prior to stimulation tests. Our model showed a good predictive power for the discrimination between subjects with and without a final GHD diagnosis, with an AUC of 0.826 .
To facilitate its clinical use, we also retrieved specific cut-offs corresponding to GHD probability of $25 \%, 50 \%$ and $75 \%$.

As previously said, GH stimulation tests are not perfect in sensitivity and specificity (19-22), and their interpretation should not therefore be viewed as a dichotomous response on patient's diagnosis. Pre-test probability estimation is of key importance to allow a proper interpretation of the test results. The presence of other pituitary deficits and the IGF-I values, evaluated in terms of agedependent SDS, have been widely recognized as predictors of GHD by many authors (34-36), as well as by current Endocrine Society guidelines (1). These results were confirmed also in our cohort, and were used to quantitatively develop a multivariate model for the estimation of GHD probability prior to stimulation tests. 


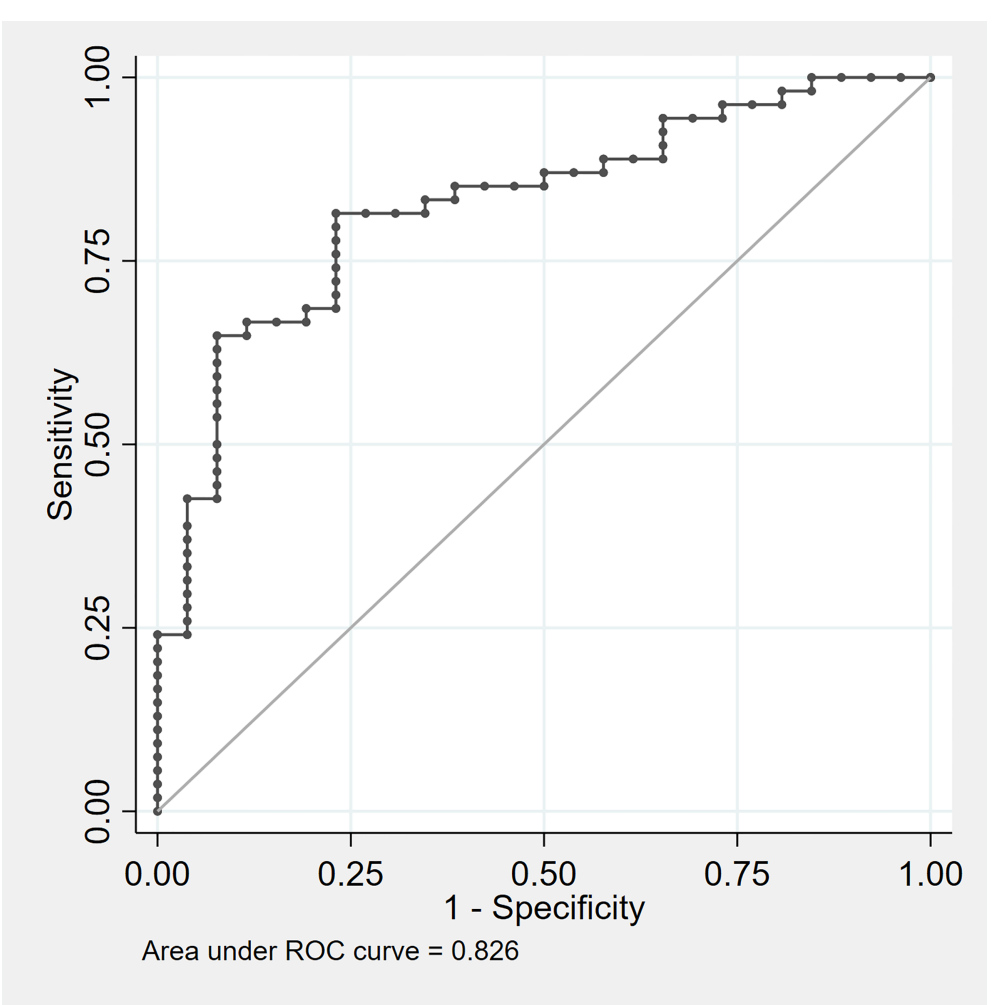

FIGURE 1 | ROC curve evaluating the diagnostic performance of the composite model predictor $z=1.82$ * other pituitary deficits (yes $=1$, no $=0$ ) -1.27 * IGF-I SDS - 1.77. ROC, receiver operating characteristic.

TABLE 3 | IGF-I SDS values predicting a pre-test GHD probability of 25\%,50\% and 75\%, stratified according to the presence or the absence of other pituitary deficits.

IGF-I SDS in the absence of other pituitary deficits

$-0.52$

$-1.39$

$-2.26$
IGF-I SDS in the presence of other pituitary deficits

+0.91
+0.04
-0.82

$-0.82$

Pre-test GHD probability

GHD, growth hormone deficiency; IGF-I, insulin-like growth factor I; SDS, standard deviation score.

The overall accuracy of the model was not sufficient to discriminate by itself between patients with and without GHD, but this was neither an expected nor a desired result. In fact, IGFI levels depend on several factors other than GH stimulation, such as nutritional status $(40,41)$ and genetic polymorphisms (42); concordantly, even if the presence/absence of other pituitary deficits can be seen as a rough approximation of the functional integrity of pituitary gland, the specific functional status of each axis is ultimately independent from that of the others.

Therefore, a provocative test should still represent a mandatory step for GHD diagnosis in most cases; our model, however, could be of significant help in clinical practice for the identification of false-positive and/or falsenegative stimulation test results. In fact, by Bayes theorem (29-31), when applying a diagnostic test characterized by approximately $90 \%$ sensitivity and specificity, if the pre-test probability of a disease or condition is $<25 \%$, the post-test probability still remains $<75 \%$ after a positive test result. Conversely, if the pre-test probability is $>75 \%$, the post-test probability still remains $>25 \%$ after a negative test result. Therefore, in patients with a low pre-test probability of GHD, a blunted GH response to one stimulation test should be interpreted with caution, and an indication for further testing should be considered; the same applies with reversed parts, i.e. in patients with a high pre-test probability of GHD and a normal GH response to one stimulation test.

We have undertaken this study with the specific aim of improving the diagnosis of adult GHD, which is currently based solely on the failure of GH response to one stimulation test. However, as already mentioned, even in the appropriate clinical context, each stimulation test has some limitations, with the possibility of false-positive and/or false-negative results. To date, the interpretation of the response to any stimulation test is based on cut-offs that do not take into account the higher or lower pre-test probability of GHD, thus ignoring a whole part of 


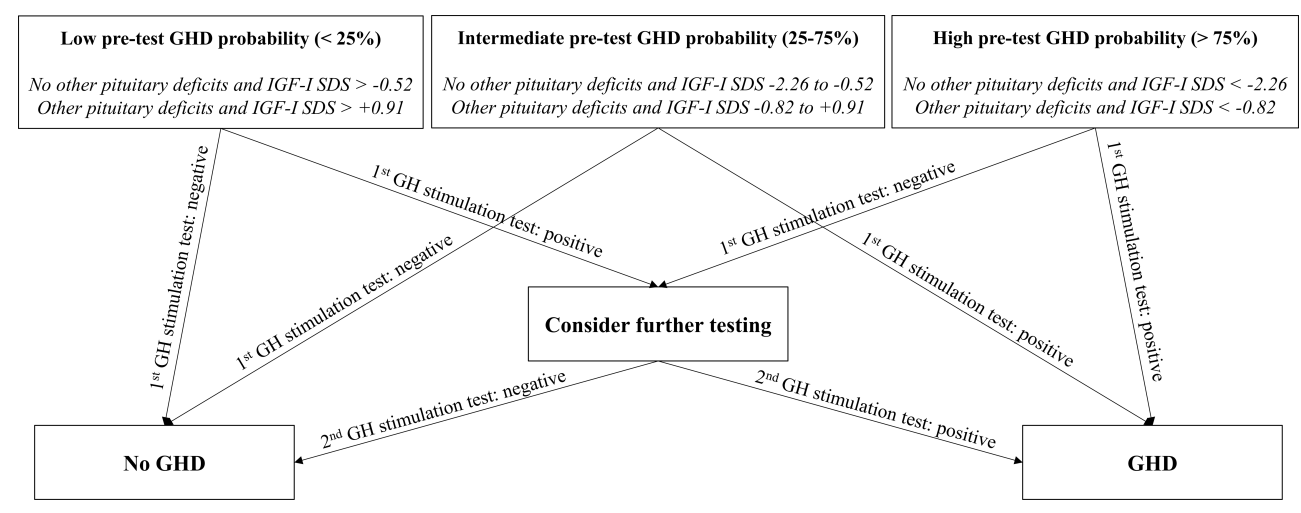

FIGURE 2 | Flow-chart proposal for integrated GHD diagnosis. GH, growth hormone; GHD, growth hormone deficiency; IGF-I, insulin-like growth factor I; SDS, standard deviation score.

complementary information which could better refine the diagnostic process. In Figure 2, therefore, we propose a flowchart for a comprehensive GHD diagnostic work-up, integrating both pre-test GHD probability estimation and stimulation test results. In line with what has just been discussed, this flow-chart suggests a specific attention in patients in which stimulation test results appear to be discordant with respect to the estimated pretest GHD probability. In these cases, a second stimulation test may be advisable for a safer confirmation or exclusion of GHD diagnosis.

The main strength of our study was the use of two recognized and concordant GH stimulation tests as the reference standard for the definition of $\mathrm{GH}$ deficiency/sufficiency, which gave a strong support to the reliability of GHD diagnosis. Moreover, the internal validation of our model conferred higher statistical consistency to the obtained results.

Our study had also some limitations. The first one was related to its retrospective design; however, the retrieved data were prospectively collected and, most notably, the recall of baseline clinical features for each patient was based only on data retrieved from clinical reports preceding the beginning of any biochemical work-up by stimulation tests. A second limitation might be related to the high prevalence of patients with GHD in the study population, which is likely a consequence of the tertiary nature of our Center; in order to mitigate the effect of class imbalance, parameter tuning was performed on balanced subsets through random undersampling of the majority class; nevertheless, the proposed model should be applied with caution in patient populations in which the prevalence of GHD differs markedly from $50 \%$.

In conclusion, this is the first study that proposes a quantitative estimation of GHD probability prior to stimulation tests. Our final flow-chart represents a simple tool that could be adopted for a Bayesian interpretation of stimulation test results, selecting patients who may benefit from a second stimulation test. The proposed approach could make a significant contribution towards the standardization of
GHD diagnostic process, and would likely result in a reduction of the risk of wrong GHD diagnoses.

\section{DATA AVAILABILITY STATEMENT}

The raw data supporting the conclusions of this article will be made available by the authors upon request, without undue reservation.

\section{ETHICS STATEMENT}

The studies involving human participants were reviewed and approved by Comitato Etico Interaziendale A.O.U. Città della Salute e della Scienza di Torino-A.O. Ordine MaurizianoA.S.L. Città di Torino. The patients/participants provided their written informed consent to participate in this study.

\section{AUTHOR CONTRIBUTIONS}

FB conceptualized the work, performed the data analysis, and contributed to the manuscript writing. VC contributed to the data collection and manuscript writing. MP-C, AMB, and NP contributed to data interpretation and manuscript writing. SG and EG supervised the manuscript drafting. VG contributed to work conceptualization, data analysis, manuscript writing, and final draft supervision. All authors contributed to the article and approved the submitted version.

\section{ACKNOWLEDGMENTS}

The authors thank Mrs. Laura Saba and all her nursing staff for their skillful technical assistance provided during the tests. 


\section{REFERENCES}

1. Molitch ME, Clemmons DR, Malozowski S, Merriam GR, Vance ML. Evaluation and Treatment of Adult Growth Hormone Deficiency: An Endocrine Society Clinical Practice Guideline. J Clin Endocrinol Metab (2011) 96:1587-609. doi: 10.1210/jc.2011-0179

2. Prodam F, Pagano L, Corneli G, Golisano G, Belcastro S, Busti A, et al. Update on Epidemiology, Etiology, and Diagnosis of Adult Growth Hormone Deficiency. J Endocrinol Invest (2008) 31:6-11.

3. Appelman-Dijkstra NM, Kokshoorn NE, Dekkers OM, Neelis KJ, Biermasz NR, Romijn JA, et al. Pituitary Dysfunction in Adult Patients After Cranial Radiotherapy: Systematic Review and Meta-Analysis. J Clin Endocrinol Metab (2011) 96:2330-40. doi: 10.1210/jc.2011-0306

4. Gasco V, Cambria V, Bioletto F, Ghigo E, Grottoli S. Traumatic Brain Injury as Frequent Cause of Hypopituitarism and Growth Hormone Deficiency: Epidemiology, Diagnosis and Treatment. Front Endocrinol (2021) 12:634415. doi: $10.3389 /$ fendo.2021.634415

5. Prodam F, Caputo M, Mele C, Marzullo P, Aimaretti G. Insights Into NonClassic and Emerging Causes of Hypopituitarism. Nat Rev Endocrinol (2021) 17:114-29. doi: 10.1038/s41574-020-00437-2

6. Cuneo RC, Salomon F, McGauley GA, Sonksen PH. The Growth Hormone Deficiency Syndrome in Adults. Clin Endocrinol (Oxf) (1992) 37:387-97. doi: 10.1111/j.1365-2265.1992.tb02347.x

7. Burman P, Mattsson AF, Johannsson G, Höybye C, Holmer H, Dahlqvist P, et al. Deaths Among Adult Patients With Hypopituitarism: Hypocortisolism During Acute Stress, and De Novo Malignant Brain Tumors Contribute to an Increased Mortality. J Clin Endocrinol Metab (2013) 98:1466-75. doi: $10.1210 /$ jc. $2012-4059$

8. Simpson H, Savine R, Sönksen P, Bengtsson BÅ, Carlsson L, Christiansen JS, et al. Growth Hormone Replacement Therapy for Adults: Into the New Millennium. Growth Horm IGF Res (2002) 12:1-33. doi: 10.1054/ ghir.2001.0263

9. Svensson J, Bengtsson BÅ, Rosén T, Odén A, Johannsson G. Malignant Disease and Cardiovascular Morbidity in Hypopituitary Adults With or Without Growth Hormone Replacement Therapy. J Clin Endocrinol Metab (2004) 89:3306-12. doi: 10.1210/jc.2003-031601

10. Jørgensen AP, Fougner KJ, Ueland T, Gudmundsen O, Burman P, Schreiner T, et al. Favorable Long-Term Effects of Growth Hormone Replacement Therapy on Quality of Life, Bone Metabolism, Body Composition and Lipid Levels in Patients With Adult-Onset Growth Hormone Deficiency. Growth Horm IGF Res (2011) 21:69-75. doi: 10.1016/j.ghir.2011.01.001

11. Widdowson WM, Gibney J. The Effect of Growth Hormone Replacement on Exercise Capacity in Patients With GH Deficiency: A Metaanalysis. J Clin Endocrinol Metab (2008) 93:4413-7. doi: 10.1210/jc.2008-1239

12. Martel-Duguech L, Jorgensen JOL, Korbonits M, Johannsson G, Webb SM, Adamidou F, et al. ESE Audit on Management of Adult Growth Hormone Deficiency in Clinical Practice. Eur J Endocrinol (2020) 184:323-34. doi: 10.1530/eje-20-1180

13. Gasco V, Prodam F, Grottoli S, Marzullo P, Longobardi S, Ghigo E, et al. GH Therapy in Adult GH Deficiency: A Review of Treatment Schedules and the Evidence for Low Starting Doses. Eur J Endocrinol (2013) 168(3):R55-66. doi: 10.1530/EJE-12-0563

14. Yuen K. Adult Growth Hormone Deficiency Guidelines: More Difficult Than it Seems to Incorporate Into Clinical Practice Universally. Eur J Endocrinol (2021) 184(4):C5-7. doi: 10.1530/EJE-20-1455

15. Vance ML. Can Growth Hormone Prevent Aging? N Engl J Med (2003) 348:779-80. doi: 10.1056/nejmp020186

16. Cook DM, Yuen KCJ, Biller BMK, Kemp SF, Vance M. American Association of Clinical Endocrinologists Medical Guidelines for Clinical Practice for Growth Hormone Use in Growth Hormone-Deficient Adults and Transition Patients - 2009 Update. Endocr Pract (2009) 15:1-29. doi: 10.4158/EP.15.S2.1

17. Ho KKY. Consensus Guidelines for the Diagnosis and Treatment of Adults With GH Deficiency II: A Statement of the GH Research Society in Association With the European Society for Pediatric Endocrinology, Lawson Wilkins Society, European Society of Endocrinology, J. Eur J Endocrinol (2007) 157:695-700. doi: 10.1530/ EJE-07-0631
18. Andersen M. The Robustness of Diagnostic Tests for GH Deficiency in Adults. Growth Horm IGF Res (2015) 25:108-14. doi: 10.1016/j.ghir.2015.03.001

19. Biller BMK, Samuels MH, Zagar A, Cook DM, Arafah BM, Bonert V, et al. Sensitivity and Specificity of Six Tests for the Diagnosis of Adult GH Deficiency. J Clin Endocrinol Metab (2002) 87:2067-79. doi: 10.1210/ jcem.87.5.8509

20. Kelestimur F, Popovic V, Leal A, Van Dam PS, Torres E, Perez Mendez LF, et al. Effect of Obesity and Morbid Obesity on the Growth Hormone (GH) Secretion Elicited by the Combined GHRH + GHRP-6 Test. Clin Endocrinol (Oxf) (2006) 64:667-71. doi: 10.1111/j.1365-2265.2006.02525.x

21. Corneli G, Di Somma C, Baldelli R, Rovere S, Gasco V, Croce CG, et al. The Cut-Off Limits of the GH Response to GH-Releasing Hormone-Arginine Test Related to Body Mass Index. Eur J Endocrinol (2005) 153:257-64. doi: 10.1530/eje.1.01967

22. Dichtel LE, Yuen KCJ, Bredella MA, Gerweck AV, Russell BM, Riccio AD, et al. Overweight/obese Adults With Pituitary Disorders Require Lower Peak Growth Hormone Cutoff Values on Glucagon Stimulation Testing to Avoid Overdiagnosis of Growth Hormone Deficiency. J Clin Endocrinol Metab (2014) 99:4712-9. doi: 10.1210/jc.2014-2830

23. Iranmanesh A, Lizarralde G, Veldhuis JD. Age and Relative Adiposity Are Specific Negative Determinants of the Frequency and Amplitude of Growth Hormone (GH) Secretory Bursts and the Half-Life of Endogenous GH in Healthy Men. J Clin Endocrinol Metab (1991) 73:1081-8. doi: 10.1210/jcem73-5-1081

24. Van Dam EWCM, Roelfsema F, Helmerhorst FH, Frölich M, Edo Meinders A, Veldhuis JD, et al. Low Amplitude and Disorderly Spontaneous Growth Hormone Release in Obese Women With or Without Polycystic Ovary Syndrome. J Clin Endocrinol Metab (2002) 87:4225-30. doi: 10.1210/ jc.2002-012006

25. Veldhuis JD, Iranmanesh A, Ho KKY, Waters MJ, Johnson ML, Lizarralde G. Dual Defects in Pulsatile Growth Hormone Secretion and Clearance Subserve the Hyposomatotropism of Obesity in Man. J Clin Endocrinol Metab (1991) 72:51-9. doi: 10.1210/jcem-72-1-51

26. Vizner B, Reiner SM. Effect of L-Dopa on Growth Hormone, Glucose, Insulin, and Cortisol Response in Obese Subjects. Exp Clin Endocrinol Diabetes (1983) 81:41-8. doi: 10.1055/s-0029-1210205

27. Cordido F, Dieguez C, Casanueva FF. Effect of Central Cholinergic Neurotransmission Enhancement by Pyridostigmine on the Growth Hormone Secretion Elicited by Clonidine, Arginine, or Hypoglycemia in Normal and Obese Subjects. J Clin Endocrinol Metab (1990) 70:1361-70. doi: 10.1210/jcem-70-5-1361

28. Gasco V, Ferrero A, Bisceglia A, Prencipe N, Cambria V, Bioletto F, et al. The Cut-Off Limits of GH Response to Insulin Tolerance Test Related to Body Mass Index for the Diagnosis of Adult GH Deficiency. Neuroendocrinology (2020) 111(5):442-50. doi: 10.1159/000508103

29. Diamond GA. The Wizard of Odds: Bayes' Theorem and Diagnostic Testing. Mayo Clin Proc (1999) 74:1179-82. doi: 10.4065/74.11.1179

30. Lesaffre E, Speybroeck N, Berkvens D. Bayes and Diagnostic Testing. Vet Parasitol (2007) 148:58-61. doi: 10.1016/j.vetpar.2007.05.010

31. Johnson KM. Using Bayes' Rule in Diagnostic Testing: A Graphical Explanation. Diagnosis Berlin Ger (2017) 4:159-67. doi: 10.1515/dx-20170011

32. Elamin MB, Murad MH, Mullan R, Erickson D, Harris K, Nadeem S, et al. Accuracy of Diagnostic Tests for Cushing's Syndrome: A Systematic Review and Metaanalyses. J Clin Endocrinol Metab (2008) 93:1553-62. doi: 10.1210/ jc.2008-0139

33. Sitch AJ, Dekkers OM, Scholefield BR, Takwoingi Y. Introduction to Diagnostic Test Accuracy Studies. Eur J Endocrinol (2021) 184:E5-9. doi: 10.1530/EJE-20-1239

34. Hartman ML, Crowe BJ, Biller BMK, Ho KKY, Clemmons DR, Chipman JJ. Which Patients do Not Require a GH Stimulation Test for the Diagnosis of Adult GH Deficiency? J Clin Endocrinol Metab (2002) 87:477-85. doi: $10.1210 /$ jcem.87.2.8216

35. Juul A, Kastrup KW, Pedersen SA, Skakkebæk NE. Growth Hormone (GH) Provocative Retesting of 108 Young Adults With Childhood-Onset GH Deficiency and the Diagnostic Value of Insulin-Like Growth Factor I (IGF-I) and IGF-Binding Protein-3 1. J Clin Endocrinol Metab (1997) 82:1195-201. doi: $10.1210 /$ jcem. 82.4 .3892 
36. Toogood AA, Beardwell CG, Shalet SM. The Severity of Growth Hormone Deficiency in Adults With Pituitary Disease Is Related to the Degree of Hypopituitarism. Clin Endocrinol (Oxf) (1994) 41:511-6. doi: 10.1111/j.13652265.1994.tb02583.x

37. Fleseriu M, Hashim IA, Karavitaki N, Melmed S, Murad MH, Salvatori R, et al. Hormonal Replacement in Hypopituitarism in Adults: An Endocrine Society Clinical Practice Guideline. J Clin Endocrinol Metab (2016) 101:3888-921. doi: $10.1210 /$ jc.2016-2118

38. Aimaretti G, Boschetti M, Corneli G, Gasco V, Valle D, Borsotti M, et al. Normal Age-Dependent Values of Serum Insulin Growthfactor-I: Results From a Healthy Italian Population. J Endocrinol Invest (2008) 5:445-9. doi: 10.1007/BF03346389

39. Krawczyk B. Learning From Imbalanced Data: Open Challenges and Future Directions. Prog Artif Intell (2016) 5:221-32. doi: 10.1007/s13748-016-0094-0

40. Keller U. Nutritional Laboratory Markers in Malnutrition. J Clin Med (2019) 8:775. doi: $10.3390 /$ jcm 8060775

41. Wang X, Tian F, Sun H, Zhang L, Gao X, Huang Y, et al. Insulin-Like Growth Factor-1 as a Nutritional Monitoring Factor in Patients With Chronic Intestinal Failure. Clin Nutr (2019) 38:1737-44. doi: 10.1016/j.clnu.2018.07.031

42. Rotwein P. Large-Scale Analysis of Variation in the Insulin-Like Growth Factor Family in Humans Reveals Rare Disease Links and Common
Polymorphisms. J Biol Chem (2017) 292:9252-61. doi: 10.1074/ jbc.M117.783639

Conflict of Interest: The authors declare that the research was conducted in the absence of any commercial or financial relationships that could be construed as a potential conflict of interest.

Publisher's Note: All claims expressed in this article are solely those of the authors and do not necessarily represent those of their affiliated organizations, or those of the publisher, the editors and the reviewers. Any product that may be evaluated in this article, or claim that may be made by its manufacturer, is not guaranteed or endorsed by the publisher.

Copyright (c) 2021 Bioletto, Parasiliti-Caprino, Berton, Prencipe, Cambria, Ghigo, Grottoli and Gasco. This is an open-access article distributed under the terms of the Creative Commons Attribution License (CC BY). The use, distribution or reproduction in other forums is permitted, provided the original author(s) and the copyright owner(s) are credited and that the original publication in this journal is cited, in accordance with accepted academic practice. No use, distribution or reproduction is permitted which does not comply with these terms. 